\title{
Pierres et châteaux, un aspect de la construction en Bourgogne au Moyen Âge (Montbard, Asnières et Brancion)
}

\section{Emmanuel Laborier et Didier Lamotte}

\author{
(2) OpenEdition \\ Journals \\ Édition électronique \\ URL : https://journals.openedition.org/cem/776 \\ DOI : $10.4000 /$ cem.776 \\ ISSN : 1954-3093 \\ Éditeur \\ Centre d'études médiévales Saint-Germain d'Auxerre \\ Édition imprimée \\ Date de publication : 15 août 2005 \\ ISSN : 1623-5770 \\ Référence électronique \\ Emmanuel Laborier et Didier Lamotte, « Pierres et châteaux, un aspect de la construction en \\ Bourgogne au Moyen Âge (Montbard, Asnières et Brancion) », Bulletin du centre d'études médiévales \\ d'Auxerre | BUCEMA [En ligne], 9 | 2005, mis en ligne le 03 novembre 2006, consulté le 22 septembre \\ 2022. URL : http://journals.openedition.org/cem/776 ; DOI : https://doi.org/10.4000/cem.776
}

Ce document a été généré automatiquement le 22 septembre 2022.

\section{(c) (i) (2)(2)}

Creative Commons - Attribution - Pas d'Utilisation Commerciale - Partage dans les Mêmes Conditions 4.0 International - CC BY-NC-SA 4.0

https://creativecommons.org/licenses/by-nc-sa/4.0/ 


\title{
Pierres et châteaux, un aspect de la construction en Bourgogne au Moyen Âge (Montbard, Asnières et Brancion)
}

\author{
Emmanuel Laborier et Didier Lamotte
}

À partir des interventions préventives menées par l'INRAP sur les châteaux de Montbard (Côte-d'Or) et de Brancion (commune de Martailly-les-Brancion, Saône-etLoire) et sur le site des carrières anciennes d'Asnières-les-Dijon, il est possible d'aborder la question de l'utilisation de la pierre dans le domaine castral et dans les constructions des ducs de Bourgogne au cours du Moyen Âge. Pour cela, il est nécessaire d'associer les observations réalisées sur les maçonneries (études d'élévation ou fouilles) et les éléments lapidaires, les données géologiques (identification, contexte naturel) et les sources d'archives médiévales et modernes (comptabilités, archives "minières"). Le croisement de ces données permet d'une part d'établir la caractérisation des roches utilisées dans la construction et d'autre part, de proposer des provenances (localisation de carrières anciennes).

2 Ce travail doit nécessairement se faire en partenariat avec des géologues pour l'identification des roches (échantillons, lames minces) et l'analyse du potentiel naturel (cartes géologiques, reconnaissance d'affleurements actuels). Ce projet s'intègre ainsi dans les axes de recherche de l'UMR 5594, dont Matériaux, techniques et sociétés (dir. Jean Rosen) et Géo-archéologie: approche des milieux, archéologie spatiale, matériaux (dir. Christophe Petit) en particulier en ce qui concerne la "géologie des matériaux". Il étudie les modes de repérage, d'évaluation et d'acquisition des matériaux, les outils d'extractions (et leurs traces archéologiques laissées sur les vestiges), l'approvisionnement des chantiers de construction et le mode de circulation des matériaux par la notion de taille et d'échelle : du local au régional et aux grandes distances et, enfin, les notions de fonctionnalité et d'ornementation des 
roches. Il participe aussi à l'identification pétrographique et à la constitution d'une lithothèque ${ }^{1}$ associée à une base de données Trans'archéo.

3 On notera que tout en étant relativement distants les uns des autres les trois sites considérés se trouvent tous dans des contextes calcaires ce qui donne une certaine homogénéité au programme de travail du point de géologique. Cependant, ces trois pôles présentent des contextes historiques et archéologiques différents. Le château de Montbard a bénéficié d'un vaste programme architectural réalisé par les ducs capétiens vers 1300 . Asnières est un centre d'extraction pour une pierre recherchée pour ses qualités de taille (sculpture entre autres). Quant au château de Brancion, il offre tout un panel d'élévations entre le $\mathrm{XI}^{\mathrm{e}}$ et le $\mathrm{XIV}^{\mathrm{e}}$ siècle avec l'emploi de matériaux visiblement différents. C'est donc dans cette pluralité que ce projet prend toute sa valeur et qu'il confère une meilleure assise aux résultats attendus.

Le château de Montbard (site du Parc Buffon)

4 Un important lot d'éléments lapidaires médiévaux, issus des interventions préventives de 1996 à 2001, est à reprendre en détail pour préciser les types de pierre utilisés en fonction du type d'élément architectural identifié. Ce lot de plus de 150 blocs et fragments est déposé pour l'essentiel au Parc Buffon et est, de par sa masse, difficilement transportable. Il convient d'en dresser un inventaire précis complété par des échantillons géologiques. Ces échantillons seront analysés au sein de l'équipe des géo-archéologues.

Montbard est un château ducal et une partie de la comptabilité est conservée entre 1340 et 1477 (Archives départementales de la Côte-d'Or, B 5300 à 5341). Des sondages dans ces documents ont montré l'intérêt de ces sources pour l'identification des carrières médiévales. Il conviendrait donc de poursuivre ces dépouillements et de les compléter par la lecture des archives récentes concernant les carrières en activité à Montbard jusqu'à une période récente. Ce travail compléterait largement la réflexion entamée lors d'un mémoire de DESS archéo-sciences (E. Laborier, 1998).Il permettrait une meilleure identification pétrographique des matériaux mis en œuvre et de proposer une carte des provenances possibles entre le $\mathrm{XIII}^{\mathrm{e}}$ et le $\mathrm{XV}^{\mathrm{e}}$ siècle. La prospection géologique et la recherche d'anciennes carrières éventuellement conservées font le lien entre les différentes phases de l'étude : étude architecturale par la présence de produits en cours d'élaboration ou finis dans les carrières, étude géologique par la reconnaissance des centres carriers et étude documentaire par les mentions des carrières ou de leurs propriétaires. Ces résultats peuvent être intégrés au programme de présentation du site du château étroitement associé au parc aménagé, au XVIII e siècle, par le naturaliste Buffon également soucieux de géologie.

Les carrières d'Asnières-les-Dijon (site du Bois des Grottes)

6 La pierre d'Asnières est un type reconnu par les géologues et identifiée sur une grande partie des collections de sculptures antiques et médiévales des musées de Dijon. Asnières fournit ainsi le grand chantier de la chartreuse de Champmol construite par le duc de Bourgogne Philippe le Hardi à la fin du XIVe siècle. Actuellement, les abords de deux anciennes carrières souterraines font l'objet d'opérations préventives qui ont déjà livré un grand nombre de blocs liés à l'extraction et à la préparation de cette pierre. Ces interventions périphériques viennent compenser l'impossibilité d'accéder directement aux carrières. Pour constituer un dossier qui permettrait l'exploitation des échantillons, différentes recherches semblent nécessaires. Il conviendrait de dépouiller la documentation récente concernant les carrières de ce secteur afin de remettre dans 
un contexte plus large les carrières souterraines concernées par nos interventions. De même, il faudrait approfondir le travail de bibliographie sur les mentions de l'utilisation de la pierre d'Asnières. Enfin, il serait intéressant de rechercher d'éventuels documents concernant les propriétaires de ces carrières souterraines au Moyen Âge. En parallèle, il faudrait réaliser un échantillonnage sur les blocs de pierre portant des traces d'outils mis au jour sur le site et sur des prélèvements réalisés dans des niveaux naturels, afin de préciser les données pétrographiques. Ainsi, à travers la lithothèque et la base de données Trans'archéo, une base de référence fiable permettrait une meilleure identification d'éléments mis en œuvre dans des bâtiments étudiés ou de blocs travaillés (sculptures) conservés dans des musées ou mis au jour en fouille. Il semblerait que ce matériau, clairement exploité à Asnières depuis l'époque médiévale, est déjà utilisé à l'époque gallo-romaine dans un rayon d'au moins $30 \mathrm{~km}$. Il convient cependant de bien faire le point sur le potentiel naturel de cette pierre et ses possibilités d'extraction pour différencier le type géologique d'Asnières du site d'extraction des carrières d'Asnières.

Le château de Brancion

7 En complément des interventions réalisées en 2003 et en 2004 et de celle débutée en 2005 sur le château par les Monuments historiques, une première approche documentaire sur les carrières du secteur paraît nécessaire auprès des Archives départementales de Saône-et-Loire. Il serait également pertinent de réaliser une première série de sondages dans la comptabilité de la châtellenie ducale de Brancion conservée entre 1347 et 1477, dans les Archives départementales de la Côte-d'Or (B 5378 à 5418), afin de connaître les lieux d'approvisionnement utilisés à cette période. Les données issues des opérations de terrain peuvent déjà nourrir la réflexion sur les différents types de pierre mis en œuvre et sur le potentiel naturel. Il est donc nécessaire que les échantillons prélevés lors des différentes interventions archéologiques soient analysés. Il sera également possible de réaliser des comparaisons avec les études d'élévations menées par Benjamin Saint-Jean Vitus sur le site proche du bourg et de l'abbaye de Tournus. Cette recherche sur les types de pierre utilisés peut permettre, en plus de la lithothèque et de la base de données Trans'archéo, fournira une référence pour le choix des matériaux employés lors des restaurations à venir par les Monuments historiques.

État de la documentation de base (non exhaustive)

- BECK P., LABORIER E., RECOUVROT C., Montbard, Parc Buffon, le Château, fouille programmée, campagne 1995, rapport, Dijon : Service Régional de l'Archéologie, 43 p.

- LABORIER E., Montbard, Parc Buffon, Le Château, plusieurs rapports de sauvetage ou de relevés archéologiques et d'architecture, de 1996 à 2002, Dijon, Service régional de l'Archéologie.

- LABORIER E., Châteaux de pierre, architecture militaire et construction en Bourgogne au XIV ${ }^{e}-\mathrm{XV}^{e} s$., mémoire de D.E.A., Université de Bourgogne, 1996, 394 p.

- LABORIER E., La pierre dans le château médiéval de Montbard, étude de matériaux de construction, mémoire de DESS archéo-sciences, Université de Bourgogne, septembre 1998, 35 p.

- LABORIER E., Asnières-les-Dijon (21), Le Bois des Grottes, lot $n^{\circ} 8$ et lot $n^{\circ} 10$, rapports de surveillance archéologique, Dijon, SRA Bourgogne, 2003 et 2004.

- LABORIER E., "Montbard, une forteresse capétienne pour les duchesses", L'art à la cour de Bourgogne, le mécénat de Philippe le Hardi et de Jean sans Peur (1634-1419), catalogue d'exposition, Musée des Beaux-Arts de Dijon, éd. RMN, Paris, 2004, p. 150-152. 
- LAMOTTE D., “Constitution d'une lithothèque : collection d'échantillons de carrières et d'échantillons archéologiques de roches utilisées pour la construction bourguignonne. Pétrographie, extraction, mise en œuvre, archives.", Études et travaux 2003-2004, Centre d'Etudes Médiévales, Auxerre, 2004, p. 169.

- LAMOTTE D., Caractérisation et origine des matériaux utilisés par les Gallo-romains d'Entrains-surNohain (58), mémoire de DESS archéo-sciences, Université de Bourgogne, septembre 1996.

- LORENZ J., TARDY D., COULON G., La pierre dans la ville antique et médiévale. Actes du colloque d'Argentomagus, Musée d'Argenton-FERAC, 2000. Avec en particulier les contributions de D. Tardy sur la méthodologie (p. 20), D. Lamotte sur un exemple d'étude (p. 75) et divers auteurs sur l'étude des archives (p. 121).

- LUKAS D., Asnières-les-Dijon “Bois des Grottes”, DFS d'évaluation archéologique, septembre 2001, Dijon, SRA Bourgogne, 2001, 18 p. + planches.

- PASCAREL, "Une carrière de marbre à Montbard", Bulletin de la Société archéologique et biographique du canton de Montbard, II, 1 ère série, 1910-1914, (1910), p. 9-11.

- RAT P., "Les pierres de l'abbatiale de Tournus, la géologie et l'homme", Société des amis des arts et sciences de Tournus, t. 95, 1996, p. 169-203.

- SAINT-JEAN VITUS B., LABORIER E., Village de Brancion, réhabilitation des voiries : projet de réseaux. Rapport de diagnostic. Dijon, Service Régional de l'Archéologie, 2003, 92 p.

- SAINT-JEAN VITUS B., LABORIER E., NOGUÈS P., SARAZIN P., Village et château de Brancion. Rapport d'étude préliminaire du potentiel archéologique. Dijon, Service Régional de l'Archéologie, 2003, 104 p.

- VICHERAT, R, "Le marbre de Buffon", Bulletin de la Société archéologique et biographique du canton de Montbard, III, janvier-juillet 1926, p. 4-5.

\section{NOTES DE FIN}

1. Cette lithothèque, initiée en 1999, suite aux travaux de Pascale Chardron-Picault (ville d'Autun) et de Didier Lamotte et plus récemment de l'équipe du PCR "Matériaux, techniques et décors architectoniques à Augustodunum : de la carrière au monument" (V. Brunet Gaston dir.- INRAP) à Autun pour la période antique, compte déjà une cinquantaine d'échantillons de roches (locales ou exotiques, archéologiques ou de carrières). Il est intéressant de remarquer que des centres carriers antiques de la région de Montbard où du Sud de la Saône-et-Loire perdurent pendant la période médiévale.

\section{INDEX}

Mots-clés : château, pierre

Index géographique : France/Montbard, France/Asnières, France/Brancion 